\title{
Estimation of stochastic environment force for master-slave robotic system
}

\author{
NEELU NAGPAL ${ }^{1, *}$, BHARAT BHUSHAN ${ }^{1}$ and VIJYANT AGARWAL ${ }^{2}$ \\ ${ }^{1}$ Department of Electrical Engineering, Delhi Technological University, Delhi 110042, India \\ ${ }^{2}$ Division of Manufacturing Processes and Automation Engineering, Netaji Subhas Institute of Technology, \\ New Delhi 110078, India \\ e-mail: nagpalneelu1971@gmail.com; bharatdce@yahoo.co.in; vijaynt.agarwal@gmail.com
}

MS received 15 August 2015; revised 10 December 2015; accepted 12 July 2016

\begin{abstract}
The aim of this work is to obtain the maximum likelihood estimate (MLE) of controller-gain parameters $\widehat{K}$ of the slave robot to determine the stochastic environment force. This is accomplished by measuring the joint positions of master and slave for a known master torque using stochastic difference equation. Here, the environmental force is modelled as a zero-mean white Gaussian random process. Therefore, the joint probability distribution function (pdf) of the slave angle over a given time duration can be computed as a function of the parameters ' $K$ '. This pdf is maximized with respect to ' $K$ ' to obtain the MLE of controller-gain parameters. Subsequently, convergence analysis of error in the estimates is performed. Also, an expression of the Cramer-Rao lower bound (CRLB) is derived to measure accuracy of the estimation. Comparison of CRLB with variance of MLE supports that our estimates are asymptotically efficient. The estimation performance is validated analytically and through simulations carried out on a two-link master-slave robotic system.
\end{abstract}

Keywords. Environmental noise; parameter estimation; trajectories; MLE; CRLB; Fisher information matrix.

\section{Introduction}

There exists a wide spectrum of controllers to tackle the potential problems related to master-slave robotic systems $[1,2]$. The problems of parameter uncertainty, time delay, disturbance, loss of information, transparency, etc. are the subject of present research and researchers have suggested various control techniques to tackle some of these problems. Model prediction adaptive controller [3], robust $\mu$ synthesis controllers $[4,5]$, intelligent controllers [6-8], nonlinear and composite adaptive controller [7, 9] and disturbance observer [10] have been used to control such dynamical systems. Further, the environment, operator and task (EOT) adaptive controllers have been considered in [11] highlighting the importance of on-line information for the control. But many times, on-line information is not available due to various limitations and the researchers have to cope with the uncertainty. The designing of controllers seeks the evaluation of environmental force as all robots ultimately perceive the world through limited and improper sensors. Mostly, environmental force has been modelled as a spring-damper system and its variants [11-16]. Another approach is to regard it as an external disturbance [17] or environmental noise and disturbance

*For correspondence observer has been suggested to estimate the environmental force. The dynamic environment effect is more realistically modelled with states of the robot and obstacle as random variables [18].

According to the central limit theorem, a large sum of small independent random effects of environmental noise converges to the Gaussian law. Therefore, in this work, environmental force has been assumed to follow the structure of Gaussian statistics. The whiteness of the environmental noise is based on the fact that usually the environmental kicks appear randomly and independent of each other, similar to a molecule that kicks a pollen particle in a fluid, leading to the pollen particle executing Brownian motion [19-22].

The technique of maximum likelihood estimation is optimal $[23,24]$ as compared with others under the condition of known trajectory. MLE works with the block processing method and acts as an efficient estimator since this estimation attains Cramer-Rao lower bound CRLB [25, 26].

Designing of a controller by considering the measured data as a joint PDF in combination with the estimation and convergence analysis of noise is not available in the literature. In this paper, this is attempted successfully. Furthermore, no work has been done on MLE of the proportional-derivative (PD) controller's coefficients of a robot, and there does not exist any computation of CRLB of the component vector comparing the matrix elements of the 
controller's gain parameters. The work presented in this paper is a novel application of MLE of nonlinear dynamics of master-slave robotics system. Moreover, the evaluation of CRLB from approximate statistics of the robot angular position and angular velocity perturbations is obtained from the correlation theory of the Gauss-Markov process.

Further, limited literature [27] exists on applications of stochastic methods in robots to model torque noise and no attempt has been made to estimate torque noise sample path trajectory from MLE.

In the proposed approach, stochastic environment force is introduced into the dynamics of slave robot, which adds formidable complexity into robotic systems dynamics. It requires reconfiguring the available $\mathrm{PD}$ controller gain parameters of master-slave robotic system by exploiting estimation methods. Hence, the joint PDF of the slave angle over a given time duration can be computed as a function of the unknown parameters $K$, where $K$ is a vector whose components are linear combination matrices of $\mathrm{PD}$ parameter entries. In this work, MLE has been proposed for estimation of controller-gain parameters; and back-substituting these controller estimates into the dynamics leads to estimation of the sample trajectory of the environmental noise process.

Convergence analysis of the parameter estimates is performed by obtaining approximate expressions for the mean square parameters estimation error based on $N$ data samples and the accuracy of environmental noise estimate is justified due to low signal to noise ratio (SNR).

Finally, an approximate expression is derived for the CRLB on the parameter estimation error covariance matrix. This lower bound sets a limit to the accuracy in estimating a parameter that influences probability density. It will be proved that as number of iterations i.e., $N \longrightarrow \infty$, the CRLB $\longrightarrow 0$, implying that efficient parameter estimates are consistent.

\section{Master-slave dynamics}

A block diagram illustrating the proposed optimal design of the PD controller's parameters and environmental noise estimation is shown in figure 1 . The slave motion does not influence the master dynamics in this model since no error feedback is provided to the master. However, the slave dynamics is determined by (a) the environmental torque, $\tau_{E}(t)$, (b) the master process, $\theta_{M}(t)$ and (c) the PD controller coefficients $K_{p}, K_{d}$, which affect the error process $e(t)$ that gets fed into the slave torque.

The inverse dynamics takes the master angles, $\theta_{m}(t)$ and angular velocities $\theta_{m}^{\prime}(t)$ as its other two variables and generates a torque $\left(\tau_{I}\right)$ that is used as input to the slave. As a result the slave tends to track the master. Assuming zero environmental torque, the PD feedback coefficients are designed based on optimal control theory to minimize the error energy between the master and slave angles for a master torque. This system is then handed over to the user, who does not know the exact values of the PD control gain parameters but wishes to use the system to determine the torque generated by a rapidly vibrating environment (environmental uncertainty). This is done by causing the slave to act on the environment and measure the angles of master and slave for a given master torque and random environmental torque $\left(\tau_{E}\right)$.

The angular measurement $\theta[$.] by the forward dynamics is being utilized to form joint pdf as a function of the unknown value of controller-gain parameters $(K)$. Maximizing the conditional pdf of the state vectors, $p\left(\theta_{s}[], K.\right)$ over a duration of time w.r.t. $K$ is equivalent to minimizing the negative likelihood function $f\left(\theta_{s}[\cdot], K\right)$. The estimated value $\widehat{K}$ is substituted in the linearized version of slave dynamics in place of $K$ to estimate the enviornment noise trajectory, $\{W[n]\}_{n=0}^{N}$.

Remark 1 The dynamic equations of both two-link master and slave robots are identical except for the input torques.

The master equation is represented as

$$
M_{m}\left(\theta_{m}\right) \theta_{m}^{\prime \prime}+N_{m}\left(\theta_{m}, \theta_{m}^{\prime}\right)=\tau_{m o}(t)
$$

where $M_{m}$ represents the moment of inertia matrix, $N_{m}$ combines the effects of damping Coriolis and centrifugal forces and gravitation and $\tau_{m o}(t)$ is torque [28]. The master equation (1) can be rearranged as

$$
\begin{gathered}
\theta_{m}^{\prime \prime}=\psi_{1}\left(\theta_{m}, \theta_{m}^{\prime}\right)+\psi_{2}\left(\theta_{m}, \theta_{m}^{\prime}\right) \tau_{m o}(t) \\
\psi_{1}\left(\theta_{m}, \theta_{m}^{\prime}\right)=-M_{m}\left(\theta_{m}\right)^{-1} N_{m}\left(\theta_{m}, \theta_{m}^{\prime}\right)
\end{gathered}
$$

and

$$
\psi_{2}\left(\theta_{m}, \theta_{m}^{\prime}\right)=M_{m}\left(\theta_{m}\right)^{-1} .
$$

For a given torque process in discrete time $\tau_{m o}[n]=\tau_{m o}[n \Delta]$ ( $\Delta$ being the discretization step size), the master robot angular position can be simulated as

$$
\begin{aligned}
&\left(\theta_{m}[n+1]-2 \theta_{m}[n]+\theta_{m}[n-1]\right) / \Delta^{2} \\
&= \psi_{1}\left(\theta_{m}[n],\left(\theta_{m}[n]-\theta_{m}[n-1]\right) / \Delta\right) \\
&+\psi_{2}\left(\theta_{m}[n],\left(\theta_{m}[n]-\theta_{m}[n-1]\right) / \Delta\right) \tau_{m o}[n+1] .
\end{aligned}
$$

The error dynamics is represented as

$$
e(t)=K_{p}\left(\theta_{m}-\theta_{s}\right)+K_{d}\left(\theta_{m}^{\prime}-\theta_{s}^{\prime}\right)
$$

The master robot controls the slave robot by inverse dynamics computation as shown in figure 1 . The inverse dynamics block takes the input as the angular position $\left(\theta_{m}\right)$ and velocity $\left(\theta_{m}^{\prime}\right)$ of master. The torque control law is computed using the angular acceleration of master $\left(\theta_{m}^{\prime \prime}\right)$ plus an error torque $(e(t))$ obtained by passing the position and velocity error through a PD controller. This error 


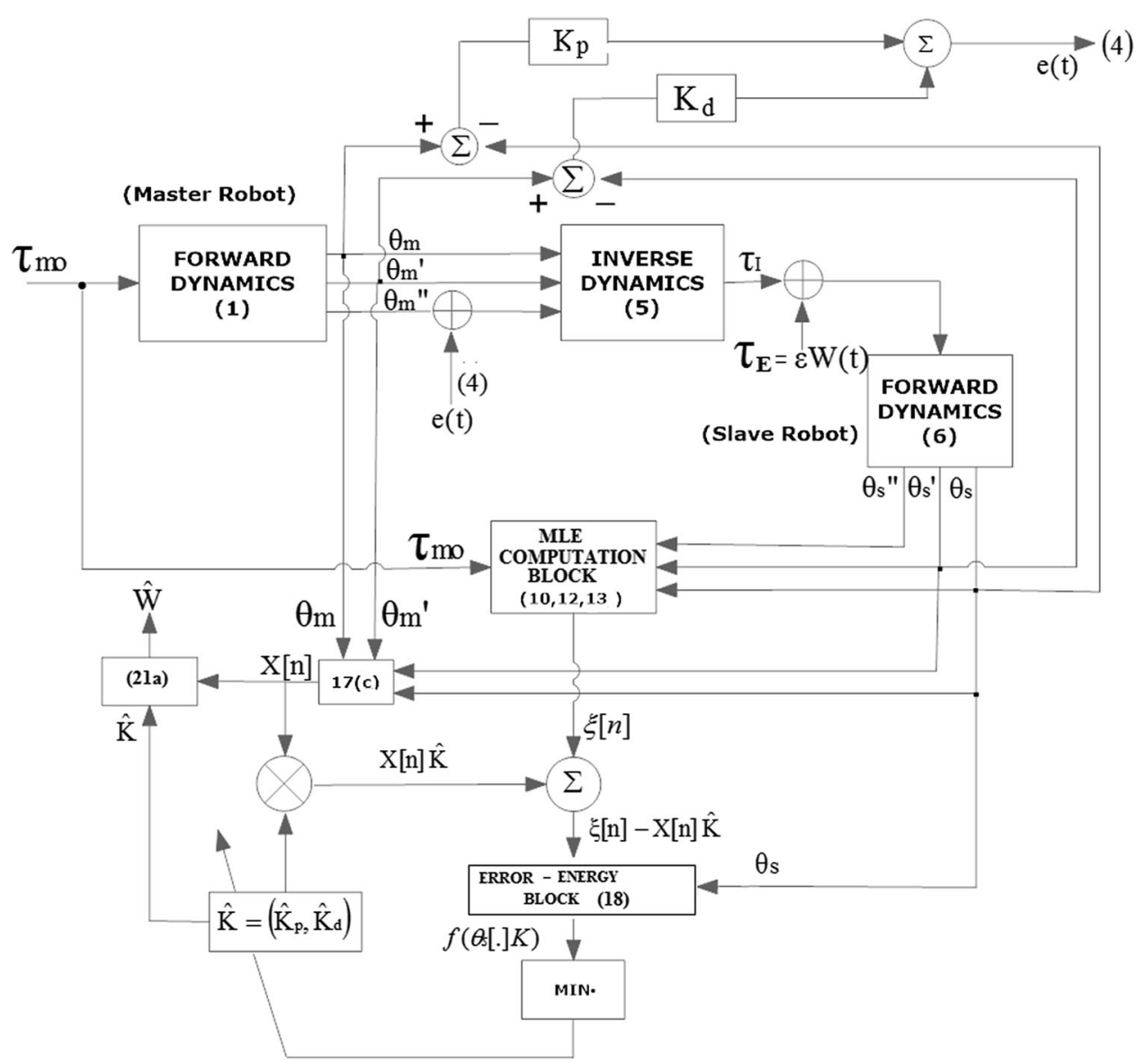

Figure 1. Block diagram for estimation process of PD controller gain parameters and environment force for master-slave system.

feedback guarantees that if the slave robot lags the master robot either in position or in velocity, then the acceleration input to the inverse dynamics block increases, thus causing the torque applied to the slave to increase so that its position and velocity approach more closely the master robot position and velocity, respectively. In the presence of environmental torque, $\tau_{E}$, and torque from inverse dynamics, $\tau_{I}$, the total torque applied to the slave robot is $\tau_{s}$. Also $\tau_{I}$ is represented as

$$
\begin{aligned}
\tau_{I}(t) & =F\left(\theta_{I}, \theta_{I}^{\prime}, \theta_{I}^{\prime \prime}\right) \\
\text { where } \theta_{I} & =\theta_{m}, \quad \theta_{I}^{\prime}=\theta_{m}^{\prime} \\
\text { and } \theta_{I}^{\prime \prime} & =\theta_{m}^{\prime \prime}+e(t)
\end{aligned}
$$

The slave equations are represented as follows:

$$
\begin{aligned}
& F\left(\theta_{s}, \theta_{s}^{\prime}, \theta_{s}^{\prime \prime}\right)=\tau_{s}=\tau_{E}+\tau_{I} \\
= & \tau_{E}+F\left(\theta_{m}, \theta_{m}^{\prime}, \theta_{m}^{\prime \prime}+e(t)\right) \\
= & \tau_{E}+F\left(\theta_{m}, \theta_{m}^{\prime}, \theta_{m}^{\prime \prime}+e(t)\right)
\end{aligned}
$$

or equivalently, the dynamics of slave robot interacting with environment is

$$
\begin{aligned}
\theta_{s}^{\prime \prime}= & -M_{s}\left(\theta_{s}\right)^{-1} N_{s}\left(\theta_{s}, \theta_{s}^{\prime}\right)+M_{s}\left(\theta_{s}\right)^{-1} M_{m}\left(\theta_{m}\right) e(t) \\
& +M_{s}\left(\theta_{s}\right)^{-1}\left(\tau_{E}+\tau_{m o}\right) \\
= & \psi_{1}\left(\theta_{s}, \theta_{s}{ }^{\prime}\right)+L\left(t, \theta_{s}, \theta_{s}{ }^{\prime}\right) e(t)+\psi_{2}\left(\theta_{s}, \theta_{s}{ }^{\prime}\right)\left(\tau_{E}+\tau_{m o}\right)
\end{aligned}
$$

where $L\left(t, \theta_{s}\right)=M_{s}\left(\theta_{s}\right)^{-1} M_{m}\left(\theta_{m}(t)\right)$.

Remark 2 Suppose $\tau_{E}$ is small, i.e., $\tau_{E}(t)=\epsilon W(t)$, where $W(t)$ is a standard 2-dimensional zero-mean white Gaussian random process and $\epsilon$ is a perturbation parameter attached to the noise term in the slave dynamics to keep track of terms having different order of smallness in the dynamics. This amounts to assuming that the noise term is of first order of smallness and enables expanding the solution in the powers of $\epsilon$. Thus the solution of the dynamical system to any degree of smallness (i.e., power of $\epsilon$ ) can be achieved by this process. At the end of the calculation, we may let $\epsilon=1$. This appears when the slave robot hits a randomly vibrating wall.

Then from Eq. (6e) and [29], the stochastic difference equation representing the discretized dynamical model of slave can be expressed as 


$$
\begin{aligned}
&\left(\theta_{s}[n+2]-2 \theta_{s}[n+1]+\theta_{s}[n]\right) / \Delta^{2} \\
&= \psi_{1}\left(\theta_{s}[n],\left(\theta_{s}[n+1]-\theta_{s}[n]\right) / \Delta\right)+L\left(n, \theta_{s}[n],\right. \\
&\left.\left(\theta_{s}[n+1]-\theta_{s}[n]\right) / \Delta\right) e[n]+\psi_{2}\left(\theta_{s}[n],\right. \\
&\left.\left(\theta_{s}[n+1]-\theta_{s}[n]\right) / \Delta\right)\left(\epsilon W[n+2] / \sqrt{\Delta}+\tau_{m o}[n+2]\right)
\end{aligned}
$$

In the above equation, $L\left[n, \theta_{s}, \theta_{s}^{\prime}\right]=L\left[n \Delta, \theta_{s}, \theta_{s}^{\prime}\right]$ and $W[n]$ is an independent and identically distributed (i.i.d.) $N\left(0, \sigma_{w}^{2} I_{2}\right)$ sequence.

Remark 3 The factor $\frac{1}{\sqrt{\Delta}}$ is attached to $W[n+2]$ is based on the fact that if $B(t)$ is standard Brownian motion [30], then $\frac{d B(t)}{d t}$ is standard white Gaussian noise and in discrete time, can be approximated by $\frac{B(t+\Delta)-B(t)}{\Delta}$, which is a Gaussian random variable having zero mean and variance $\frac{1}{\Delta}$.

Equation (7) can be arranged as

$$
\begin{aligned}
\theta_{s}[n+2]= & 2 \theta_{s}[n+1]-\theta_{s}[n]+\Delta^{2} \psi_{1}\left(\theta_{s}[n],\left(\theta_{s}[n+1]-\theta_{s}[n]\right) / \Delta\right) \\
& +\Delta^{2} L\left(n, \theta_{s}[n],\left(\theta_{s}[n+1]-\theta_{s}[n]\right) / \Delta\right)\left(K_{p}\left(\theta_{m}[n]-\theta_{s}[n]\right)\right. \\
& +\left(K_{d} / \Delta\right)\left(\theta_{m}[n+1]-\theta_{m}[n]-\theta_{s}[n+1]+\theta_{s}[n]\right) \\
& +\psi_{2}\left(\theta_{s}[n],\left(\theta_{s}[n+1]-\theta_{s}[n]\right) / \Delta\right) \\
& \times\left(\epsilon \Delta^{3 / 2} W[n+2]+\Delta^{2} \tau_{m o}[n+2]\right) .
\end{aligned}
$$

\section{Estimation of controller's gain parameters with stochastic environmental Force}

\subsection{Controller design}

It is assumed that designing of controller parameters, i.e., $K_{p}$ and $K_{d}$ is done so that $\left\{\sum_{n=0}^{N-1}\left\|\theta_{m}[n]-\theta_{s}[n]\right\|^{2}\right\}$ is a minimum in the presence of noise. Direct minimization of $\left\{\sum_{n=0}^{N-1}\left\|\theta_{m}[n]-\theta_{s}[n]\right\|^{2}\right\}$ w.r.t. $K_{p}, K_{d}$ is equivalent to a maximum likelihood problem since the noise has been considered to be white Gaussian. This design can be achieved using stochastic optimal control methods like Bellman's stochastic dynamic programming method.

Remark 4 Having designed $K_{p}$ and $K_{d}$, noise is introduced through the environment in the slave dynamics and the robot is handed over to a user who does not know values of gain parameters and the environment. This user takes measurements of noisy slave position $\delta \theta_{s}[n]=\theta_{s}[n]-$ $\theta_{m}[n]$ over a finite time duration; then using MLE, he estimates the unknown controller's gain parameters $K_{p}$ and $K_{d}$ and after that estimates to determine the environmental noise.
The aim is to design $K_{p}$ and $K_{d}(2 \times 2$ matrices $)$ so that

$$
\begin{aligned}
& \mathbb{E}\left\{\sum_{n=0}^{N-1}\left\|\theta_{m}[n]-\theta_{s}[n]\right\|^{2}\right\} \text { is minimum, } \\
& \text { where } \theta_{s}[n]-\theta_{m}[n] \approx \delta \theta_{s}[n] \text { is of } O(\epsilon) .
\end{aligned}
$$

After linearizing Eq. (8) about $\theta_{s}^{(0)}[n]=\theta_{m}[n]$, the linear time-varying stochastic difference equation is represented as

$$
\begin{aligned}
\delta \theta_{s}[n+2]= & 2 \delta \theta_{s}[n+1]-\delta \theta_{s}[n] \\
& +\Delta^{2} \Psi_{1,1}[n] \delta \theta_{s}[n]+\Delta \Psi_{1,2}[n]\left(\delta \theta_{s}[n+1]-\delta \theta_{s}[n]\right) \\
& -\Delta^{2} L[n]\left(K_{p} \delta \theta_{s}[n]+K_{d} / \Delta\left(\delta \theta_{s}[n+1]-\delta \theta_{s}[n]\right)\right) \\
& +\Delta^{2} \Psi_{2,1}[n]\left(\delta \theta_{s}[n] \otimes \tau_{m o}[n+2]\right) \\
& \left.+\Delta \Psi_{2,2}[n]\left(\delta \theta_{s}[n+1]-\delta \theta_{s}[n]\right) \otimes \tau_{m o}[n+2]\right) \\
& +\Delta^{3 / 2} \Psi_{2}[n] W[n+2] .
\end{aligned}
$$

It may be noted that

$$
\begin{aligned}
& \psi_{1}[n]=\psi_{1}\left(\theta_{m}[n], \theta_{m}^{\prime}[n]\right) \\
& \psi_{2}[n]=\psi_{1}\left(\theta_{s}[n], \theta_{s}^{\prime}[n]\right)
\end{aligned}
$$

where $\psi_{1,1}[n]=\frac{\partial \psi_{1}\left(\theta_{m}[n], \partial \theta_{m}^{\prime}[n]\right)}{\left(\partial \theta_{s}[n]\right)}$.

$$
\text { Also, } \theta_{m}^{\prime}[n]=\frac{\theta_{m}[n]-\theta_{m}[n-1]}{\Delta} \text {. }
$$

Similarly, $\theta_{s}^{\prime}[n]=\frac{\theta_{s}[n]-\theta_{s}[n-1]}{\Delta}$

$$
\begin{aligned}
& \psi_{1,2}[n]=\frac{\partial \psi_{1}\left(\theta_{m}[n], \partial \theta_{m}^{\prime}[n]\right)}{\left(\partial \theta_{s}[n]\right)} \\
& \psi_{2,1}[n]=\frac{\partial \psi_{2}\left(\theta_{m}[n], \partial \theta_{m}^{\prime}[n]\right)}{\left(\partial \theta_{s}^{\prime}[n]\right)} \\
& \psi_{2,2}[n]=\frac{\partial \psi_{2}\left(\theta_{m}[n], \partial \theta_{m}^{\prime}[n]\right)}{\left(\partial \theta_{s}^{\prime}[n]\right)} .
\end{aligned}
$$

$\theta_{m}[n]=\theta_{m}[n \Delta]$ is obtained by sampling the continuous time master trajectory.

$\theta_{s}[n]=\theta_{s}[n \Delta]$ is obtained by sampling the continuous time slave trajectory.

Also, $L[n]=L\left(n, \theta_{m}[n],\left(\theta_{m}[n+1]-\theta_{m}[n]\right) / \Delta\right)=I_{2}$.

Applying elementary linear algebra techniques involving Kronecker product in Eq. (10), this can be derived as

$$
\begin{aligned}
\delta \theta_{s}[n+2]= & {\left[2 I_{2}+\Delta \Psi_{1,2}[n]-\Delta K_{d}\right.} \\
& \left.\left.+\Delta \Psi_{2,2}[n]\left(I_{2} \otimes \tau_{m o}\right)[n+2]\right)\right] \delta \theta_{s}[n+1] \\
& +\left[I_{2}+\Delta^{2} \Psi_{1,1}[n]-\Delta \Psi_{1,2}[n]-\left(\Delta^{2} K_{p}-\Delta K_{d}\right)\right. \\
& +\Delta \Psi_{2,1}[n]\left(I_{2} \otimes \tau_{m o}[n+2]\right)-\Delta \Psi_{2,2}[n] \\
& \left.\times\left(I_{2} \otimes \tau_{m o}[n+2]\right)\right] \delta \theta_{s}[n]+\Delta^{3 / 2} \Psi_{2}[n] W[n+2] .
\end{aligned}
$$


We can define

$$
\begin{aligned}
\Delta K_{d}= & \alpha_{d} \in \mathbb{R}^{2 \times 2}, \quad \Delta^{2} K_{p}-\Delta K_{d}=\beta_{d} \in \mathbb{R}^{2 \times 2} \\
A[n]= & 2 I_{2}+\Delta \Psi_{1,2}[n]+\Delta \Psi_{2,2}[n]\left(I_{2} \otimes \tau_{m o}[n+2]\right) \\
B[n]= & -I_{2}+\Delta^{2} \Psi_{1,1}[n]-\Delta \Psi_{1,2}[n]+\Delta \Psi_{2,1}[n] \\
& \times\left(\tau_{m o}[n+2] \otimes I_{2}\right)-\Delta \Psi_{2,2}[n]\left(I_{2} \otimes \tau_{m o}[n+2]\right)
\end{aligned}
$$

$$
C[n]=\Delta^{5 / 2} \Psi_{2}[n] .
$$

Then Eq. (11) can be rearranged as

$$
\begin{aligned}
\delta \theta_{s}[n+2]= & {\left[A[n]-\alpha_{d}\right] I_{2} \delta \theta_{s}[n+1] } \\
& +\left[B[n]-\beta_{d}\right] I_{2} \delta \theta_{s}[n]+C[n] W[n+2] .
\end{aligned}
$$

This equation is now in the right form required for applying the ML method to estimate $\alpha_{d}$ and $\beta_{d}$ (or equivalently $K_{p}$ and $\left.K_{d}\right) \cdot\left\{\delta \theta_{s}[n]\right\}$ follows 2 nd order difference equation with white-noise-driving term [31].

The pdf of $\left\{\delta \theta_{s}[n]\right\}$ parameterized by $\alpha_{d}$ and $\beta_{d}$ can be calculated by the following process based on second order Markov model [32]:

$$
\begin{aligned}
& p\left(\delta \theta_{s}[n+2] \mid \delta \theta_{s}[n+1], \delta \theta_{s}[n]\right) \\
&=1 / 2 \pi|C[n]| \exp \left\{-1 / 2 \sigma^{2}\left(\delta \theta_{s}[n+2]-\left(A[n]-\alpha_{d}\right) \delta \theta_{s}[n+1]\right.\right. \\
&-\left(B[n]-\beta_{d}\right)\left|\delta \theta_{s}[n]\right|^{T}\left(C[n] C[n]^{T}\right)^{-1} \delta \theta_{s}[n+2] \\
&\left.-\left(A[n]-\alpha_{d}\right) \delta \theta_{s}[n+1]-\left(B[n]-\beta_{d}\right) \delta \theta_{s}[n]\right\} \\
&= 1 /|C[n]| \Phi\left(C [ n ] ^ { - 1 } \left(\delta \theta_{s}[n+2]-\left(A[n]-\alpha_{d}\right)\right.\right. \\
&\left.\left.\delta \theta_{s}[n+1]-\left(B[n]-\beta_{d}\right) \delta \theta_{s}[n]\right)\right) ; \\
& \text { where } \Phi(\xi)=\frac{1}{2 \pi} \exp \left(-\|\xi\|^{2} / 2\right), \quad \xi \in \mathbb{R}^{2} .
\end{aligned}
$$

Thus the ML estimates of $\alpha_{d}$ and $\beta_{d}$ based on the measurements of $\left\{\delta \theta_{s}[n]: 0 \leq n \leq N\right\}$ are given by setting the variational derivative of the product of all terms in Eq. (14) over $0 \leq n \leq N$ w.r.t. $\alpha_{d}$ and $\beta_{d}$ to zero and assuming $P[n]=\left(C[n] C[n]^{T}\right)^{-1}$. The resulting expression can be arranged in the following form using elementary properties of the Kronecker tensor product, especially $\operatorname{Vec}(A \times B)=$ $\left(B^{T} A\right) \otimes \operatorname{Vec}(X)[33]:$

$$
\begin{aligned}
\operatorname{Vec} & \left\{\sum_{n} P[n] \xi[n+2] \delta \theta_{s}[n+1]^{T}\right\} \\
& \left.+\left(\sum_{n}\left(\delta \theta_{s}[n+1] \delta \theta_{s}[n+1]^{T}\right) \otimes P[n]\right) \operatorname{Vec}\left(\widehat{\alpha}_{d}\right)\right) \\
& \left.+\left(\sum_{n}\left(\delta \theta_{s}[n+1] \delta \theta_{s}[n]^{T}\right) \otimes P[n]\right) \operatorname{Vec}\left(\widehat{\beta}_{d}\right)\right)=0
\end{aligned}
$$

$$
\text { and } \begin{aligned}
\operatorname{Vec} & \left\{\sum_{n} P[n] \xi[n+2] \delta \theta_{s}[n]^{T}\right\} \\
& +\left(\sum_{n}\left(\delta \theta_{s}[n] \delta \theta_{s}[n+1]^{T} \otimes P[n]\right) \operatorname{Vec}\left(\widehat{\alpha}_{d}\right)\right) \\
& +\left(\sum_{n}\left(\delta \theta_{s}[n] \delta \theta_{s}[n]^{T} \otimes P[n]\right) \operatorname{Vec}\left(\widehat{\beta}_{d}\right)\right)=0 .
\end{aligned}
$$

Equations (16a) and (16b) form a set of eight simultaneous linear equations for the unknown parameter vector $\left[\begin{array}{l}\operatorname{Vec}\left(\alpha_{d}\right) \\ \operatorname{Vec}\left(\beta_{d}\right)\end{array}\right] \in \mathbb{R}^{8}$.

They can be expressed as follows and can be inverted immediately:

$$
\begin{aligned}
& {\left[\begin{array}{cc}
\sum \delta \theta_{s}[n+1] \delta \theta_{s}[n+1]^{T} \otimes P[n] & \sum \delta \theta_{s}[n+1] \delta \theta_{s}[n]^{T} \otimes P[n] \\
\sum \delta \theta_{s}[n] \delta \theta_{s}[n+1]^{T} \otimes P[n] & \sum \delta \theta_{s}[n] \delta \theta_{s}[n]^{T} \otimes P[n]
\end{array}\right]} \\
& \quad \times\left[\begin{array}{c}
\operatorname{Vec}\left(\widehat{\alpha}_{d}\right) \\
\operatorname{Vec}\left(\widehat{\beta}_{d}\right)
\end{array}\right] \\
& =-\left[\begin{array}{c}
\operatorname{Vec}\left(\sum P[n] \xi[n+2] \delta \theta_{s}[n+1]^{T}\right) \\
\operatorname{Vec}\left(\sum P[n] \xi[n+2] \delta \theta_{s}[n]^{T}\right)
\end{array}\right] .
\end{aligned}
$$

Equation (16c) can be solved for the $4 \times 1$ vectors, i.e., $\operatorname{Vec}\left(\widehat{\alpha}_{d}\right)$ and $\operatorname{Vec}\left(\widehat{\beta}_{d}\right)$, i.e.,

$$
K=\left[\begin{array}{l}
\operatorname{Vec}\left(\alpha_{d}\right) \\
\operatorname{Vec}\left(\beta_{d}\right)
\end{array}\right] \text { or equivalently for the } 8 \times 1 \text { vector. }
$$

The proposed maximum likelihood estimator of $K$, i.e., $\widehat{K}[N]$ based on slave linearization, has been expressed in the form $K+P^{-1} Q$ where $Q$ is a bilinear vector function of $\left\{\delta \theta_{s}[n]\right\}$ and $\{W[n]\}$, and $P$ is a quadratic function $\delta \theta_{s}[n]$. Here, $\left\{\delta \theta_{s}[n]\right\}$ is the master-slave angular error. In view of the linearized dynamics, $P$ and $Q$ are both quadratic functions of the environmental torque noise $W[n]$. Thus $\widehat{K}-$ $K=\left\|\mathbb{E} P^{-1}\right\|^{2}\|\mathbb{E} Q\|^{2}$ can be regarded as a "matrix ratio" of two quadratic functions of $W[n]$. The mean value of $Q$ turns out to be zero in view of the system causality. The mean value of $P$ is non-zero, and hence if approximation of $\mathbb{E}(\widehat{K}-K)$ by $\left(\mathbb{E} P^{-1}\right)(\mathbb{E} Q)$ is done, result is zero. This justifies the PD estimator of the proposed work being nearly unbiased. Also, approximating $\mathbb{E}\left[\|\widehat{K}[n]-K\|^{2}\right]$ by $\|$ $(\mathbb{E} P) \|^{2} \mathbb{E}\left[\|Q\|^{2}\right]$, it can be proven that this is small using the fact that $Q$ is a sum of independent $\chi^{2}$ random variables. We then substitute the estimated value $\widehat{K}[N]$ of $K$ into the slave dynamics(non-linear) in place of $\mathrm{K}$, and solve for $W[n]$ in terms of $\{\theta[n]\}_{n=0}^{N}$ and $\widehat{K}[n]$. The result is $\{W[n]\}_{n=0}^{N}$, an estimate of the environmental torque process. Then it can be shown that $\widehat{W}[n]-W[n]$ is a bilinear 
function of $\delta \theta_{s}[n]$ and $\{\widehat{K}[n]-K\}$ and is therefore of second order of smallness, i.e., of $O\left(\|W\|^{2}\right)$. This justifies our accurate estimate of the environmental torque $\widehat{W}[n]$, from which the structure of the environment can be determined, and computations of this are presented in the following section.

\subsection{Environment noise estimation}

Using the general nonlinear dynamical model of slave given in Eq. (8), the aim is to compute the statistics of $(\widehat{K}[N]-K)$ and $(\widehat{W}[N]-W)$. We now present in brief the ML parameter estimates based on the exact nonlinear dynamics. However, for computation of the performance of these estimates, it is required that we linearize the dynamics otherwise the evaluation of the angular correlation becomes impossible. The expression given in Eq. (7) can also be written in the linear form as

$$
\xi[n+2]=X[n] K+Y[n] W[n+2]
$$

where

$$
\begin{gathered}
\xi[n+2]=\theta_{s}[n+2]-2 \theta_{s}[n+1]+\theta_{s}[n] \\
-\Delta^{2} \Psi_{1}\left(\theta_{s}[n] \theta_{s}[n+1)-\theta_{s}[n] / \Delta\right. \\
-\Delta^{2} \Psi_{1}\left(\theta_{s}[n] \tau_{m o}[n+2]\right) \\
X[n]=-\Delta^{2} L\left[n, \theta_{s}[n]\right]\left(\delta q[n]^{T} \otimes I_{2}\right) \\
Y[n]=\epsilon \Delta^{3 / 2} \psi_{2}\left(\theta_{s}[n]\right)
\end{gathered}
$$

Remark 5 Recall that:

$$
\begin{aligned}
L\left[n, \theta_{s}[n]\right] & \left.=M_{s}\left(\theta_{s}[n]\right)^{-1} M_{m}[n]\right), \\
\delta q[n] & =\left[\begin{array}{c}
\delta \theta_{s}[n] \\
\delta \omega_{s}[n]
\end{array}\right], \\
\delta \theta_{s}[n] & =\theta_{s}[n]-\theta_{m}[n] \\
\delta \omega_{s}[n] & =\omega_{s}[n]-\omega_{m}[n] .
\end{aligned}
$$

Based on the model (17a), we can immediately write down the ML estimate of $K$ based upon samples up to time $N+2$.

Remark 6 After linearization, $L\left[n, \theta_{s}[n]\right]=I_{2}$ since $\theta_{s}$ $\approx \theta_{m}$ and $M_{m} \approx M_{s}$

$$
\begin{aligned}
\widehat{K}[N]= & \operatorname{argmin}_{K} \sum_{n=0}^{N}\left\|Y^{-1}[n](\xi[n+2]-X[n] K)\right\|^{2} \\
= & {\left[\sum_{n=0}^{N} X[n]^{T}\left(Y[n] Y[n]^{T}\right)^{-1} X[n]\right]^{-1} } \\
& {\left[\sum_{n=0}^{N} X[n]^{T}\left(Y[n] Y[n]^{T}\right)^{-1} \xi[n+2]\right] }
\end{aligned}
$$

$$
=K+H[N]^{-1} \sum_{n=0}^{N} F[n] W[n+2]
$$

where

$$
\begin{gathered}
H[N]=\sum_{n=0}^{N} X[n]^{T}\left(Y[n] Y[n]^{T}\right)^{-1} X[n] \\
F[n]=X[n]^{T}\left(Y[n] Y[n]^{T}\right)^{-1} Y[n]
\end{gathered}
$$

Up to $O\left(\|\delta q\|^{2}\right)$ in numerator and denominator, $(W[n]=O(\|\delta q\|))$, we then have $\widehat{K}[N]-K=P^{-1} Q$ as

$$
\begin{aligned}
\widehat{K}[N]-K= & \lambda\left(\sum_{n=0}^{N}\left(\delta q[n] \otimes I_{2}\right) R[n]^{-1}\left(\delta q[n]^{T} \otimes I_{2}\right)\right)^{-1} \\
& \left(\sum_{n=0}^{N}\left(\delta q[n] \otimes I_{2}\right) R[n]^{-1} \psi_{2}[n] W[n+2]\right) \\
& \text { where } \lambda=\Delta^{-4} x \omega\left(-\Delta^{2}\right) x \in \Delta^{3 / 2}=-\epsilon \Delta^{-1 / 2},
\end{aligned}
$$

$$
\begin{aligned}
R[n] & =\Psi_{2}\left(\theta_{m}[n]\right) \Psi_{2}\left(\theta_{m}[n]\right)^{T}=\Psi_{2}[n] \Psi_{2}[n]^{T} \\
\text { and } \Psi_{2}[n] & =\Psi_{2}\left(\theta_{m}[n]\right) .
\end{aligned}
$$

From Eqs. (18c) and (18d), the following is expressed as

$$
\begin{aligned}
& H[n] \propto \sum_{0}^{N}\left(\delta q[n] \otimes I_{2}\right) R[n]^{-1}\left(\delta q[n]^{T} \otimes I_{2}\right) \\
& \text { and linearization is around } \theta_{m}[n],
\end{aligned}
$$

$$
\text { Also, } \sum_{0}^{N} F[n] W[n+2] \propto \sum_{0}^{N}\left(\delta q[n] \otimes I_{2}\right) R[n]^{-1} \psi_{2}[n] W[n+2] \text {. }
$$

Remark 7 The numerator in Eq. (19) has mean zero since $\delta q[n]$ is uncorrelated with $W[n+2]$ and denominator has non-zero mean since $\mathbb{E}\left[\delta q[n] \delta q[n]^{T}\right] \neq 0$. Hence $\widehat{K}[N]-K$ is small in probability. This justifies our model.

The noise estimate from Eq. (19) is estimated as

$$
\begin{gathered}
\widehat{W}[n+2]=Y[n]^{-1}(\xi[n+2]-X[n] \widehat{K}[N]) \\
=Y[n]^{-1}(X[n] K+Y[n] W[n+2]-X[n] \widehat{K}[N]) .
\end{gathered}
$$

\section{Convergence analysis}

The aim is to determine the limiting mean square deviation $\lim _{n \uparrow 0} \mathbb{E}\left\{\left\|\delta \theta_{s}[n]\right\|^{2}\right\}, \delta \theta_{s}[n]=\theta_{s}[n]-\theta_{m}[n]$ of the slave position, which requires linearization of the nonlinear slave dynamics in discrete time about the master position. The linearized equation has time-dependent matrix coefficients 
and a driving white noise term coming from the environmental torque, which provides evaluation of correlation $\mathbb{E}\left\{\delta \theta_{s}[n] \delta \theta_{s}[n]^{T}\right\}$. Aiming that the master has a limiting angular position, derivation of a linear algebraic equation for the limiting error correlation matrix as a function of the feedback parameters $K_{p}$ and $K_{d}$ is done.

The limiting NSR is represented as $\frac{\mathbb{E}\left[\left\|\delta \theta_{s}(\infty)\right\|^{2}\right]}{\left\|\delta \theta_{m}(\infty)\right\|^{2}}$.

Approximate formulas for the PD parameter error $\widehat{K}[n]-$ $K$ and the noise estimation error $\widehat{W}[n]-W[n]$ in terms of $\mathrm{W}[\mathrm{n}] \quad$ and $\left.\quad \delta q[n]=\left(\theta_{s}[n]-\theta_{m}[n]\right), \omega_{s}[n]-\omega_{m}[n]\right) \quad$ are obtained. Specifically, the expression for the approximate value of $\widehat{K}[n]-K$ is evaluated as a ratio of two quadratic functions of a Gaussian process, i.e., $\widehat{K}[n]-K=$ $P^{-1} Q$, where $Q \in 8 \times 1$ is a quadratic function of $\delta q[n]$. We show that $Q$ has zero mean and $P$ has non-zero mean and this justifies our approach that $\mathbb{E}\{\widehat{K}[n]-K\}$ is small. A recursive algorithm is derived for calculating the correlation $\mathbb{E}\left[\delta q[n] \delta q[n]^{T}\right]$ and $\mathbb{E}\left[\delta q[n] W\left[m^{T}\right]\right.$ using linearized version of the dynamical model and this enables us to perform the above calculations.

The noise estimate error energy is given by

$$
\begin{aligned}
\widehat{W}[n+2]-W[n+2]= & Y[n]^{-1} X[n](K-\widehat{K}[N]) \\
\approx & \epsilon^{-1} \Delta^{-3 / 2} \Psi_{2}[n]^{-1}\left(-\Delta^{2}\right)\left(\delta q[n]^{T}\right. \\
& \left.\otimes I_{2}\right)(K-\widehat{K}[N]) \\
= & -\epsilon^{-1} \sqrt{\Delta} \Psi_{2}[n]^{-1}\left(\delta q[n]^{T} \otimes I_{2}\right) \\
& (K-\widehat{K}[N])
\end{aligned}
$$

The approximate expression for the absolute error $\mid \widehat{W}[n]-$ $W[n] \mid$ is obtained as a bilinear function of $\delta q[n]$ and $\{\widehat{K}[n]-K\}$, showing that this is small in probability.

This analysis is based on substituting $\widehat{K}$ in place of $K$ in Eq. (21a) and $\widehat{W}[n+2]$ in place of $W[n]$ in the same equation and substituting the resulting equation from Eq. (21b).

Since, in Eq. (22), $\widehat{W}[n+2]-W[n+2]$ is proportional to the tensor product of two small quantities $\delta q[n]$ and $K-$ $\widehat{K}[N], \widehat{W}[n+2]-W[n+2]$ is very small in probability. Thus an environmental noise estimate is highly accurate as also demonstrated in the simulations.

Remark 8 The random variables $\{\delta q[n] \otimes W[n+2]\}, n=$ $0,1,2, \ldots$ are uncorrelated owing to the whiteness of $W($.) and hence the variance of the numerator in Eq. (22) grows with $N$ as $\mathrm{O}(N)$. Equivalently, the mean modulus of the numerator is of $O(\sqrt{N})$. On the other hand, since $\mathbb{E}\left(\delta q[n] \delta q[n]^{T}\right) \neq 0$, the mean modulus of the denominator in Eq. (22) grows as $\mathrm{O}(N)$. Hence the mean modulus of

$$
\begin{aligned}
& \text { Eq. (22) decreases } \\
& \frac{O(\sqrt{N})}{O(N)}=O\left(\frac{1}{\sqrt{(N)}}\right)
\end{aligned}
$$

with increasing $N$

as

By considering the linearized model, the expression for the state equation for $q[n]$ is as a linear first-order difference equation with the white Gaussian input. This readily verifies the Gaussianity of $\delta q[n]$; standard methods can be used to derive recursive equations for its covariance evolution for calculating $\mathbb{E}\|\widehat{K}[N]-K\|^{2}$ using Eq. (19) can be done.

\section{CRLB of estimation}

The significance of obtaining the CRLB for controller-gain parameters estimates is that, since the estimates $\widehat{K_{p}}$ and $\widehat{K_{d}}$ are substituted in Eq. (22) to get an estimate of the environment noise process, these uncertainties in $\widehat{K_{p}}$ and $\widehat{K_{d}}$ will reflect as an error in the noise estimate $\widehat{W}-W$. Thus, the minimum variance in the noise estimate $\mathbb{E}\left(\|\widehat{W}-W\|^{2}\right)$ taken over all estimates of $\widehat{K_{p}}$ and $\widehat{K_{d}}$ will be lower bounded by a quantity proportional to the CRLB for $K_{p}$ and $K_{d}$.

Evaluation of the CRLB for any unbiased estimator of the estimation problem under consideration requires the use of a linearized model. This is computed as a linear combination of the master-slave error angular position-velocity correlation matrix.

Remark 9 The diagonal entries of the CRLB determine the minimum possible variance/mean square estimation error $\mathbb{E}(K-\widehat{K}[N])^{2}$ of each component of $K[N]$. The angular position-velocity correlation matrix approximately satisfies a linear difference equation of slave dynamics and by solving this equation recursively, the Fisher information matrix and hence the CRLB is obtained.

Concrete approximations of the CRLB that give its order of magnitude are derived by assuming that the master angular position has stabilized as a constant value. In this case, the linearized slave dynamics is a linear time-invariant state variable system of the form

$$
\begin{aligned}
\delta q[n+1] & =A[K] \delta q[n]+G W[n+2], \text { where } \delta q \\
& =\left[\delta \theta_{s}, \delta \omega_{s}\right]^{T} .
\end{aligned}
$$

This state variable equation is easily solved using the state transition matrix in discrete time and an expression for the error angular position-velocity correlation matrix $R_{q}[n]=$ $\left\{\delta q[n] \delta q[n]^{T}\right\}$ is immediately obtained.

Remark 10 For stability, we assume that all the eigenvalues of $A[K]$ have magnitude smaller than unity and then we derive, using the spectral norm, a bound on $\left\|R_{q}[n]\right\|$ in 
term of the maximum eigenvalue magnitude of $A[K]$. This enables us to derive the order of magnitude of the CRLB and hence of the parameter variance estimate. We show that the CRLB $\longrightarrow 0$ as $N \longrightarrow \infty$, implying that efficient parameter estimates are consistent.

The expression for the Fisher inverse matrix (i.e., the CRLB) that is obtained in the special case when the robot vibrates noisily around a fixed point.

Computation of Fisher information matrix is presented as

$$
\begin{aligned}
& J(K)=-\mathbb{E} \frac{\delta^{2} \log }{\delta K \delta K^{T}} p(q(.) \mid K) \\
& =\sum_{n=0}^{N} \mathbb{E} X[n]^{T}\left(Y[n] Y[n]^{T}\right)^{-1} X[n](\text { see Eq. (17)) } \\
& \approx \epsilon^{-2} \Delta^{-3} \Delta^{4} \sum_{n=0}^{N} \mathbb{E}\left(\delta q[n] \otimes I_{2}\right) R[n]^{-1}\left(\delta q[n]^{T} \otimes I_{2}\right) \\
& =\epsilon^{-2} \Delta \sum_{n=0}^{N}\left[\begin{array}{cc}
\delta q[n] & 0 \\
0^{T} & \delta q[n]
\end{array}\right]\left[\begin{array}{ll}
\left(R[n]^{-1}\right)_{11} & \left(R[n]^{-1}\right)_{12} \\
\left(R[n]^{-1}\right)_{21} & \left(R[n]^{-1}\right)_{22}
\end{array}\right]\left[\begin{array}{cc}
\delta q[n]^{T} & 0 \\
0^{T} & \delta q[n]^{T}
\end{array}\right] \\
& =\epsilon^{-2} \Delta \sum_{n=0}^{N}\left[\begin{array}{ll}
\left(R[n]^{-1}\right)_{11} \mathbb{E}\left\{\delta q[n] \delta q[n]^{T}\right\} & \left(R[n]^{-1}\right)_{12} \mathbb{E}\left\{\delta q[n] \delta q[n]^{T}\right\} \\
\left(R[n]^{-1}\right)_{21} \mathbb{E}\left\{\delta q[n] \delta q[n]^{T}\right\} & \left(R[n]^{-1}\right)_{22} \mathbb{E}\left\{\delta q[n] \delta q[n]^{T}\right\}
\end{array}\right] \\
& =\epsilon^{-2} \Delta \sum_{n=0}^{N}\left(R[n]^{-1} \otimes R_{q}[n]\right), \text { where } R_{q}[n] \\
& =\mathbb{E}\left\{\delta q[n] \delta q[n]^{T}\right\} \\
& \text { and the CRLB of } K \text { is } J(K)^{-1} \\
& =\frac{\epsilon^{2}}{\Delta}\left(\sum_{n=0}^{N} R[n]^{-1} \otimes R_{q}[n]\right)^{-1} .
\end{aligned}
$$

Remark 11 The diagonal entries of $J(K)^{-1} \in R^{2 \times 2}$ give a lower bound for the variance of the corresponding entry of $\widehat{K}[N]$. For evaluating the CRLB, $J(K)^{-1}$, we require $R_{q}[n]$, which has been calculated in Appendix A.

Remark 12 If we assume that the master angle vector $\theta_{m}[n]$ has stabilized around some nominal value, say $\theta_{m}^{*}$, then $\Psi_{1,1}[n]$ and $\Psi_{1,2}[n]$ are nearly constant matrixes, namely $\Psi_{1,1}\left(\theta_{m}^{*}, o\right)$ and $\Psi_{1,2}\left(\theta_{m}^{*}, o\right)$ and so is $\psi_{2}[n]=\psi_{2}\left(\theta_{m}^{*}\right)$.

Then $A[n, k]=A[K]$ is a constant matrix and so is $G[n]=$ $G$ (say).

In this case we have $\delta q[n+1]=A[K] \delta q[n]+G \omega[n+2]$, the solution to which is obtained after assuming $\delta q[0]=0$ ) and $\delta q[n]=\sum_{k=0}^{n-1} A[k]^{n-k-l} G W[k+2]$. Then, $R_{q}[n]=$ $\mathbb{E}\left[\delta q[n] \delta q[n]^{T}\right]=\sum_{k=0}^{n-1} A[K]^{n-k-l}\left|G G^{T}\left(A[K]^{T}\right)^{n-k-l}\right|$ and this can be used to get an approximate expression for the CRLB.

$$
\begin{aligned}
G & \approx \epsilon \sqrt{\Delta}\left[\begin{array}{c}
o \\
\psi_{2}
\end{array}\right] \\
G G^{T} & =\epsilon^{2} \Delta\left[\begin{array}{cc}
o & o \\
o & \psi_{2} \psi_{2}^{T}
\end{array}\right]=\epsilon^{2} \Delta\left[\begin{array}{ll}
o & o \\
o & R
\end{array}\right] .
\end{aligned}
$$

Assumption Let $A[k]=\sum_{\alpha=1}^{4} \lambda_{\alpha} \vartheta_{\alpha} \vartheta_{\alpha}^{T}$ be the spectral representation of $A[k]$.

Then,

$$
\begin{gathered}
R_{q}[n]=\epsilon^{2} \Delta \sum_{k=0}^{n-1} \sum_{\alpha, \beta=1}^{4}\left(\lambda_{\alpha} \lambda_{\beta}\right)^{n-k-1} \vartheta_{\alpha} \vartheta_{\alpha}^{T}\left[\begin{array}{cc}
0 & 0 \\
0 & R
\end{array}\right] \vartheta_{\beta} \vartheta_{\beta}^{T} \\
=\epsilon^{2} \Delta \sum_{\alpha, \beta=1}^{4}\left(\frac{1-\left(\lambda_{\alpha} \lambda_{\beta}\right)^{n}}{1-\lambda_{\alpha} \lambda_{\beta}}\right) \vartheta_{\alpha} \vartheta_{\alpha}^{T}\left[\begin{array}{cc}
0 & 0 \\
0 & R
\end{array}\right] \vartheta_{\beta} \vartheta_{\beta}^{T} \\
\text { As } n \rightarrow \infty, \text { it can be reduced to } \\
=\epsilon^{2} \Delta \sum_{\alpha, \beta=1}^{4}\left(\frac{1}{1-\lambda_{\alpha} \lambda_{\beta}}\right) \vartheta_{\alpha} \vartheta_{\alpha}^{T}\left[\begin{array}{cc}
0 & 0 \\
0 & R
\end{array}\right] \vartheta_{\beta} \vartheta_{\beta}^{T} \\
\text { Assuming }\left|\lambda_{\alpha}\right|<1 \forall \alpha, \text { from Eq. }(23 \mathrm{~g}), \text { we get, } \\
\text { CRLB }=J^{-1} \approx \frac{1}{\Delta^{2} N} R^{-1} \otimes \sum_{\alpha, \beta=1}^{4}\left(\frac{1}{1-\lambda_{\alpha} \lambda_{\beta}}\right) \\
\vartheta_{\alpha} \vartheta_{\alpha}^{T}\left[\begin{array}{ll}
(0 & 0 \\
0 & R
\end{array}\right] \vartheta_{\beta} \vartheta_{\beta}^{T} \\
\text { Then } R_{q}[n]=\epsilon^{2} \Delta \sum_{k=0}^{n-1} A[K]^{n-k-1}\left[\begin{array}{ll}
(0 & 0 \\
0 & R
\end{array}\right] A^{T}[K]^{n-k-1} \\
\text { and CRLB }(K)=\frac{1}{\Delta^{2}}\left[\sum_{n=0}^{N} R^{-1} \otimes \sum_{k=0}^{n-1}\right. \\
\left.\left(A[K]^{n-k-1}\left[\begin{array}{ll}
(0 & 0 \\
0 & R
\end{array}\right] A^{T}[K]^{n-k-1}\right)\right]^{-1} \cdot
\end{gathered}
$$

Remark 13 In Eq. (24), for a stable linearized system, eigenvalues of $A[k]$ will all be smaller than unity in magnitude and hence the inner sum will be upper bounded by a finite positive definite matrix, say $P$, as $n \rightarrow \infty$ and subsequently as $N \rightarrow \infty$, the $\operatorname{CRLB}(K)$ will converge to zero as

$$
\operatorname{CRLB}(K) \approx \frac{1}{N \Delta^{2}}\left(R^{-1} \otimes P\right)^{-1}=\frac{1}{N \Delta^{2}}\left(R \otimes P^{-1}\right)
$$

\section{Simulation results}

For simulation purpose the moment of inertia matrix of a unit length planner two-link manipulator (both master and slave) is

$$
\mathbf{M}=\left[\begin{array}{cc}
\cos \left(q_{1}-q_{2}\right)+5 / 3 & \cos \left(q_{1}-q_{2}\right)+1 / 3 \\
\cos \left(q_{1}-q_{2}\right)+1 / 3 & 1 / 3
\end{array}\right]
$$

We have taken PD gain parameters matrices as 

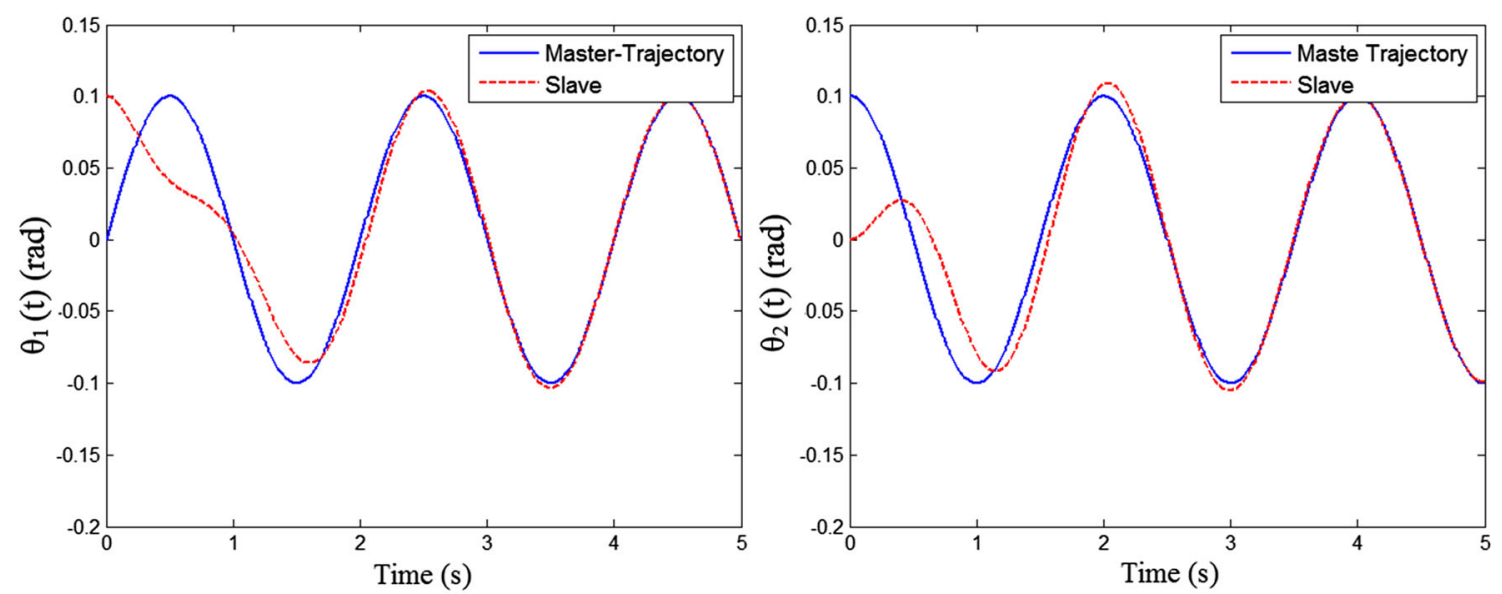

Figure 2. Trajectories of joint 1 and joint 2 of the Master-Slave System.
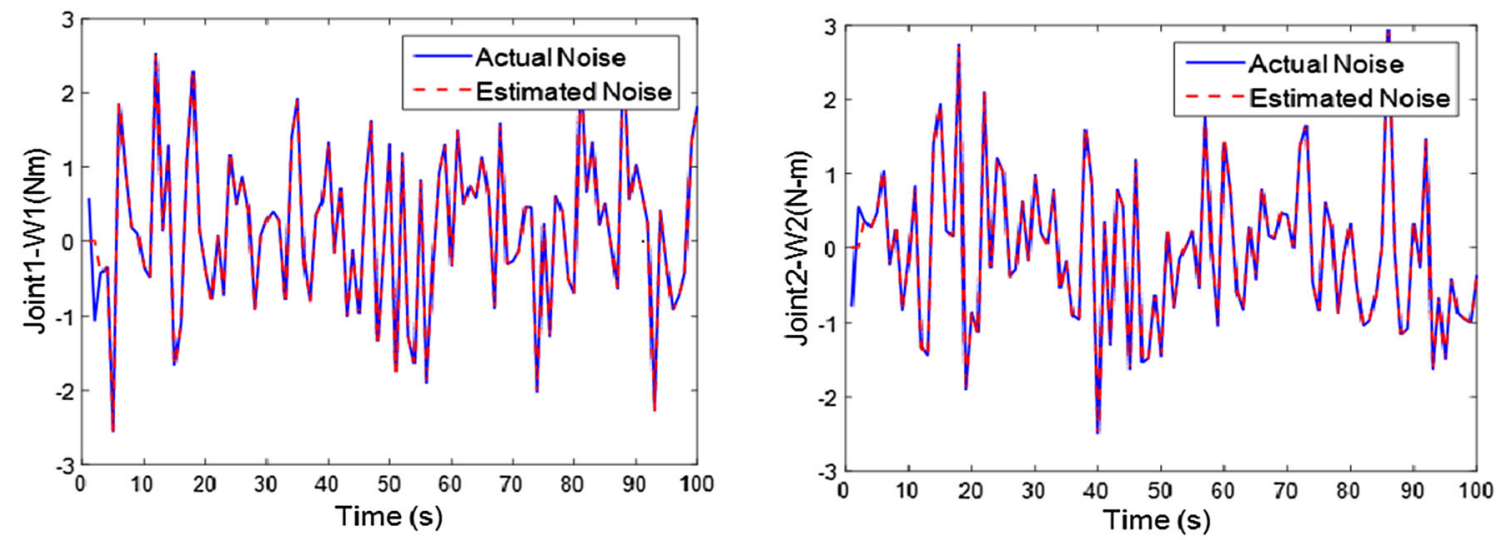

Figure 3. Estimation performance of environment noise of joint 1 and joint 2 of Slave robot.

$$
\alpha_{d}=\left[\begin{array}{cc}
20 & 0.01 \\
0.01 & 20
\end{array}\right] \text { and } \beta_{d}=\left[\begin{array}{cc}
2 & 0.01 \\
0.01 & 2
\end{array}\right] .
$$

Then we add random environmental noise with 0.01 covariance. The deviation $\theta_{s}[n]$ of the slave angles due to this noise is measured and using this deviation, the estimation of $\alpha_{d}$ and $\beta_{d}$ using MLE (from section 3.1) is

$$
\widehat{\alpha_{d}}=\left[\begin{array}{cc}
19.99 & -0.1 \\
0.4 & 20
\end{array}\right] \text { and } \widehat{\beta_{d}}=\left[\begin{array}{cc}
1.85 & 0.06 \\
-0.1 & 1.8
\end{array}\right] \text {. }
$$

With this PD controller's gain parameters, tracking plots of the slave robot are depicted in figure 2.

Error plot of this trajectory (figure 3 ) illustrates that error converges with the proposed technique.

Figure 4 illustrates that estimated environment force accurately tracks the actual environmental random force.

\section{Conclusion}

This paper has presented the algorithm of estimating environment force using MLE based on measurements of the slave position process over a given time range. The performance of parameter estimation has been explored through formulation of analytical expression of convergence and CRLB.

The success of algorithm is exemplified through convergence analysis that provides low noise to signal ratio for the parameter estimates as number of samples $(N)$ increases. Computation of CRLB of parameter estimation has been carried out that indicates that as $N$ tends to infinity, the CRLB variance approaches zero, which means that our parameter estimates are consistent.

The expressions for the parameter and noise estimation errors show via intuitive arguments that they are small in probability and decrease with increasing data length. The simulation results show the effectiveness of the estimation and encourage our contribution to recast the approach of conventional control in tracking of master-slave robotics system.

\section{Acknowledgements}

The authors acknowledge the discussion with Professor Harish Parthasarathy, Netaji Subhas Institute of 


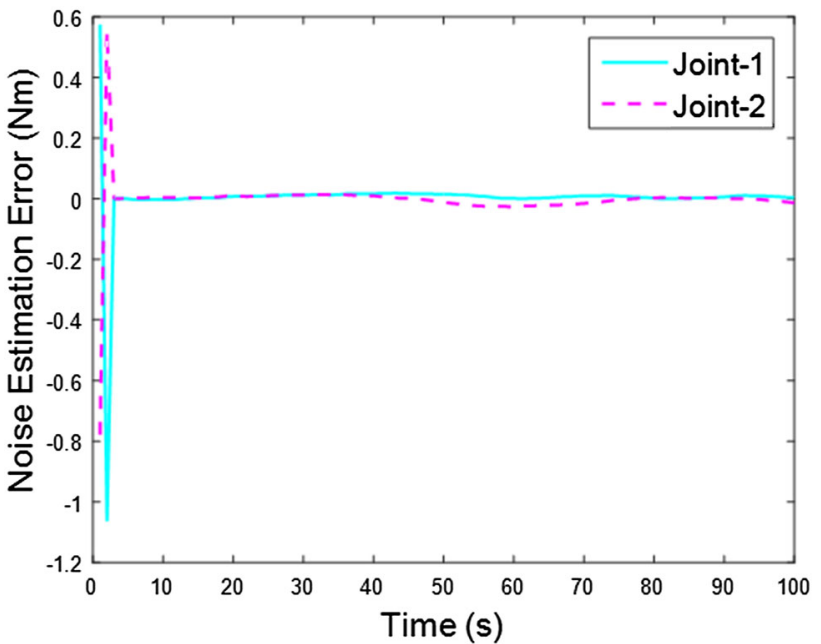

Figure 4. Time histories of estimation error of environment noise of Master-Slave system.

Technology, Delhi, India, who has helped in improving the quality of this paper.

\section{Appendix}

Computation of the statistics of $\delta q[n]$

$$
\begin{gathered}
\text { Define } \delta \theta_{s}[n]=\theta_{s}[n]-\theta_{m}[n], \\
\delta \omega_{s}[n]=\omega_{s}[n]-\omega_{m}[n] \\
\delta q[n]=\left[\begin{array}{c}
\delta \theta_{s}[n] \\
\delta \omega_{s}[n]
\end{array}\right]
\end{gathered}
$$

The state equations satisfied by $\delta q[n]$ are

$$
\begin{aligned}
& \delta \theta_{s}[n+2]-2 \delta \theta_{s}[n+1]+\delta \theta_{s}[n]=\Delta^{2}\left(\Psi_{1,1}[n] \delta \theta_{s}[n]+\Psi_{1,2}[n] \delta \omega_{s}[n]\right) \\
& \quad-\Delta^{2}\left(\left(\delta \theta_{s}[n]^{T}, \delta \omega_{s}[n]^{T}\right) \otimes I_{2}\right) K+\epsilon \Delta^{3 / 2} \Psi_{2}[n] W[n+2]
\end{aligned}
$$$$
\text { or } \delta \theta_{s}[n+1]=\delta \theta_{s}[n]+\Delta \delta \omega_{s}[n], \delta \omega_{s}[n+1]
$$$$
=\delta \omega_{s}[n]+\Delta\left(\Psi_{1,1}[n] \delta \theta_{s}[n]+\Psi_{1,2}[n] \delta \omega_{s}[n]\right)
$$$$
-\Delta\left(\left(\delta \theta_{s}[n]^{T}, \delta \omega_{s}[n]^{T}\right) \otimes I_{2}\right) K
$$$$
+\epsilon \sqrt{\Delta} \Psi_{2}[n] W[n+2] \text {. }
$$

Note that $\left(\eta^{T} \otimes I\right) \operatorname{Vec}(B)=B \eta$

Write $K$ as the $2 \times 4$ matrix formed from $K=\left[K_{p} \mid K_{d}\right]$, where $K_{p}$ and $K_{d}$ are $2 \times 2$ matrices. We thus have

$$
\begin{aligned}
\delta \omega_{s}[n+1]= & \delta \omega_{s}[n]+\Delta\left(\Psi_{1,1}[n] \delta \theta_{s}[n]+\Psi_{1,2}[n] \delta \omega_{s}[n]\right) \\
& -\Delta K_{p} \delta \theta_{s}[n]-\Delta K_{d} \delta \omega_{s}[n] \\
& +\epsilon \sqrt{\Delta} \Psi_{2}[n] W[n+2]
\end{aligned}
$$

$$
\begin{aligned}
\text { or } & {\left[\begin{array}{c}
\delta \theta_{s}[n+1] \\
\delta \omega_{s}[n+1]
\end{array}\right] } \\
= & {\left[\begin{array}{cc}
I_{2} & \Delta I_{2} \\
\left(\delta \Psi_{1,1}[n]-\Delta K_{p}\right) & \left(I+\Delta \Psi_{1,2}[n]-\Delta K_{d}\right)
\end{array}\right]\left[\begin{array}{c}
\delta \theta_{s} \\
\delta \omega_{s}
\end{array}\right] } \\
& +[\epsilon \sqrt{\Delta}]\left[\begin{array}{c}
O \\
\Psi_{2}[n]
\end{array}\right] W[n+2] .
\end{aligned}
$$$$
\text { Putting } A[n, K]=\left[\begin{array}{cc}
I_{2} & \Delta I_{2} \\
\left(\delta \Psi_{1,1}[n]-\Delta K_{p}\right) & \left(I+\Delta \cdot \Psi_{1,2}[n]-\Delta K_{d}\right)
\end{array}\right] \text { and }
$$$$
G[n]=\epsilon \sqrt{\Delta}\left[\begin{array}{c}
O \\
\Psi_{2}[n]
\end{array}\right]
$$

Equation (26b) can be expressed as

$$
\begin{gathered}
\delta q[n+1]=A[n, K] \delta q[n]+G[n] W[n+2] . \\
\text { Let } \mathbb{E}\left[\delta q[n] \delta q[n]^{T}\right]=R_{q}[n] .
\end{gathered}
$$

Then $R_{q}[n+1]=A[n, k] R_{q}[n] A^{T}[n, k]+G[n] G[n]^{T}$.

$$
\text { Also } \mathbb{E}\left[\delta q[n] W[m]^{T}\right]=0 .
$$

$$
\mathbb{E}\left[\delta q[n] W[m]^{T}\right]=A[n, k] \mathbb{E}\left[\delta q[n] W[m]^{T}\right], \quad n \geq m-1
$$

$$
\begin{aligned}
\mathbb{E}\left[\delta q[m-1] W[m]^{T}\right] & =\mathbb{E}\left[\delta q[n+1] W[n+2]^{T}\right] \quad(28 \mathrm{f}) \\
\mathbb{E}\left[\delta q[n+1] W[n+2]^{T}\right] & =A[n, k] \mathbb{E}\left[\delta q[n] W[n+2]^{T}\right]+G[n]
\end{aligned}
$$

Note that, $\delta q[n]$ is a Gaussian vector sequence and its correlation is determined from the above equations.

\section{References}

[1] Hokayem P F and Spong M W 2006 Bilateral teleoperation: an historical survey. Automatica 42(12): 2035-2057

[2] Islam S, Liu P X and El Saddik A 2013 Nonlinear control for teleoperation systems with time varying delay. Nonlinear Dyn. 76(2): 931-954

[3] Sirouspour S and Shahdi A 2006 Model predictive control for transparent teleoperation under communication time delay. IEEE Trans. Robot. 22(6): 1131-1145

[4] Polushina I G, Tayebib A and Marquezc H J 2006 Control schemes for stable teleoperation with communication delay based on IOS small gain theorem. Automatica 42: 905-915

[5] Namerikawa T, Ito S and Kawada H 2005 Robust control of master-slave robot system considering environment uncertainties. Proc. SPIE 60520F-1

[6] Agarwal V 2012 Trajectory planning of redundant manipulator using fuzzy clustering method. Int. J. Adv. Manuf. Technol. 61(528): 727-744 
[7] Li Z and Su CY 2013 Neural-adaptive control of singlemaster multiple-slaves teleoperation for coordinated multiple mobile manipulators with time-varying communication delays and input uncertainties. IEEE Trans. Neural Netw. Learn. Syst. 24(9): 1400-1413

[8] Watcharin P-N 2013 Adaptive four-channel neuro-fuzzy control of a master-slave robot. Int. J. Adv. Robot. Syst. 10: 150. doi:10.5772/55591

[9] Kim B Y and Ahn H S 2013 A design of bilateral teleoperation systems using composite adaptive controller. Control Eng. Pract. 21: 1641-1652

[10] Mohammadi A, Tavakoli M and Marquez H J 2011 Disturbance observer-based control of non-linear haptic teleoperation systems. IET Control Theory Appl. 5(18): 2063-2074

[11] Passenberg C, Peer A and Buss M 2010 A survey of environment-, operator-, and task adapted controllers for teleoperation systems. Mechatronics 20: 787-801

[12] Cho C W S 2000 Nonlinear random vibration, analytical techniques and applications, 2nd ed. Boca Raton, FL: CRC Press

[13] Erickson D, Weber M and Sharf I 2003 Contact stiffness and damping estimation for robotic systems. Int. J. Robot. Res. 22(1): 41-57

[14] Hua C C and Liu P X 2009 Convergence analysis of teleoperation systems with unsymmetric time-varying delays. IEEE Trans. Circuits Syst. II: Express Briefs 56(3): 240244

[15] Love L J and Book W J 2004 Force reflecting teleoperation with adaptive impedance control. IEEE Trans. Syst. Man Cybern. B Cybern. 34(1): 159-165

[16] Yamamoto T, Bernhardt M, Peer A, Buss M and Okamura A M 2008 Techniques for environment parameter estimation during telemanipulation. In: Proceedings of the 2nd Biennial IEEE/RAS-EMBS International Conference on Biomedical Robotics and Bio mechatronics, USA

[17] Chan L, Naghdy F and Stirling D 2013 Extended active observer for force estimation and disturbance rejection of robotic manipulators. Robot. Auton. Syst. 61(12): 1277-1287

[18] Vitus M P and Tomlin C J 2011 Belief space planning for linear, Gaussian systems in uncertain environments. In: 18th World Congress of the International Federation of Automatic Control, Milan, Italy

[19] Van Trees H L, Bell K L and Tian Z 2013 Detection, estimation and modulation theory, part I: detection, estimation, and filtering theory, 2nd ed. London: Wiley, ISBN: 978-0470-54296-5

[20] Rao P B L S 1980 Statistical inference for stochastic processes. London: Academic Press

[21] Rao R C 2001 Linear statistical interference and its applications, 2nd ed. London: Wiley

[22] Singla R, Agarwal V and Parthasarathy H 2015 Statistical analysis of tracking and parametric estimation errors in a 2-link robot based on Lyapunov function. Nonlinear Dyn. 82(1): 217-238

[23] Jazwinski A H 1970 Stochastic processes and filtering theory. New York: Academic Press, ISBN 0-12-381550-9

[24] Myung I J 2002 Tutorial on maximum likelihood estimation. J. Math. Psychol. 47(2003): 90-100

[25] Moschitta A and Carbone P 2007 Noise parameter estimation from quantized data. IEEE Trans. Instrum. Meas. 56(3): 736-742

[26] Saatci E and Akan A 2010 Inverse modeling of respiratory system during noninvasive ventilation by maximum likelihood estimation. EURASIP J. Adv. Signal Process. 2010: 237562. doi:10.1155/2010/237562

[27] Phong L D, Choi J, Lee W and Kang S 2015 A novel method for estimating external force: simulation study with a 4-DOF robot manipulator. Int. J. Precis. Eng. Manuf. 16(4): 755-766

[28] Spong M W, Hutchinson S and Vidyasagar M 2005 Robot modelling and control, 1st ed., London: Wiley

[29] Mendel J M 1987 Lessons in digital estimation theory. Prentice Hall Signal Processing Series

[30] Rao P B L S 2002 Nonparametric inference for a class of stochastic partial differential equations based on discrete observations. Indian J. Stat. 64(Ser A, pt. 1): 1-15

[31] Ioannis K and Shreve S 1998 Brownian motion and stochastic calculus. Graduate texts in Mathematics, vol. 113, 2nd ed. 470 pp. New York: Springer, ISBN 978-1-46120949-2

[32] Smith L 2006 Sequential Monte Carlo particle filtering for state estimation. Thesis, Carleton University, Ottawa, Ontario

[33] Brewer J 1998 Kronecker products and matrix calculus in system theory. IEEE Trans. Circuits Syst. 25(9): 772-781 